\title{
Risk Management of Occupational Health and Safety in Rice Farmers in Ngrendeng, East Java in 2012
}

\author{
Anisa Yonelia, L. Meily Kurniawidjaja \\ Occupational Health and Safety Department, Universitas Indonesia, Depok, 16424, Indonesia \\ E-mail: anisa.yonelia91@gmail.com;meily.widjaja@gmail.com
}

\begin{abstract}
Agriculture is an important sector that has big impact to the society thus a program that can keep its sustainability is needed, especially in term of productivity. The focus of this study was risk management of Occupational Health and Safety (OHS) in rice farmers in Ngrendeng Village, Ngawi, East Java in 2012 with analyze hazard and risk on activities and workplaces. This study was a semi-quantitative analitical descriptive with observational approach using Australian Standard/New Zealand Standard (AS/NZS) 4360:2004 about risk management. Analysis was made based on whole farming processes observed and information from village headman, a land and farm machines owner, and 5 farmers. The result showed that rice farmers' activities have 71 risks from 16 work activities and hazard sources. Rice grinding activity was a high risk activity with 11 risks. The highest value of risk, reaching 1500 (very high), comes from ergonomy, in which noise exposure, vibration exposure, UV radiation exposure, chemical exposure from pesticide and fertilizer, gas exposure, and dust exposure attained. In brief, rice farming was an agriculture activity with high risks that harmful for farmers' health and safety. Therefore, OHS program would be needed to manage hazard and risk and keep farmers' productivities.
\end{abstract}

Keywords — Risks; Rice Farmers; Risk Management

\section{INTRODUCTION}

The number of workers in the world have been increasing every year. International Labour Organization (ILO) showed that there was an increase in the number of labour, reaching 52 millions between 2004 to 2007 and 33 million between 2008 to 2011. In line with that fact, Indonesia workers has increased to 109.670 .399 in 2011 from 93.722.036 in 2004 (BPS, 2012). Most of that number comes from agricultural sector, reaching 39.328 .915 or $35,86 \%$ of all workers in 2011 (BPS, 2012). However, the number of farmers decreased by $5,2 \%$ from 41,49 million in 2010 to 39,33 million (HKTI, 2012). That was caused by labour shortages that usually comes from family relation.

Agricultural Census in 2003 showed that most of farmer families ( $45 \%$ to $85 \%$ ) have 3 to 4 family members. Moreover, decreasing number of farmer was caused by lack of interest from young man to become farmer because lack of interesting incentive as result of low product price, higher input price, none of land distribution, and narrowing of farming land. The average of land ownership was 0,5 hectares per householder. This was lower than ideal land ownership, that is 2 hectares (Sumarno and Kartasasmita, 2009).Those are the reason why farmers start seeking more incomes by cultivating more lands and keeping the production with agricultural mechanization. Thus, Occupational Health and Safety (OHS) risks were raised to high-level risk. In Great Britain, Fatal Injury Rate in agricultural (include horticulture, forestry, and other sector) reached 8 per 100.000 workers in 2010-2011. Moreover, Major Injury from farm activities reached 242,1 per 100.000 workers in 2009-2010. Farmers also suffered Work Related Diseases, such as zoonosis infections, Musculoskeletal Disorders (MSDs), Hand Arm Vibration, Vibration WhiteFinger, Whole-Body Vibration, and respiratory diseases (HSE, 2012). In 2010, there are 476 fatalities in farming with fatality rate 26,1 per 100.000 workers in USA. Farmers suffered pesticide related diseases, pulmonary diseases, Musculoskeletal Disorders (MSDs), hearing loss, and stress (NIOSH, 2011).

The high number of workers and high of fatality rate in agricultural sector had to be alert the authority to create prevention programs for various of work related diseases and accidents, and increasing workers' productivity which will have significant impact to farm sustainability and global market competition.

Indonesia rice production in 2011 can be considered high, reaching 68.061.715 tons. Main rice producer areas are West Java, East Java, and Central Java. However, production reduction number of East Java would be higher in 2012 that would increase by 11,69 million tons. One of the main 
producer areas in East Java is Ngawi Residence. Thus, Ngawi has to sustain and increase its farmers' productivity to comply with that target by implementing OHS program. Therefore, analysis about hazard and risk in farmers activities would be needed as basis to organize OHS program in agricultural. The objective of this research was to analyze OHS hazard and risk in rice farmers in Ngrendeng, Ngawi, East Java in 2012.

\section{Methodology}

\section{A. Samples}

Research subject was hazard and risk in rice farmers activities in Ngrendeng, Ngawi. Information about hazard and risk was procured from observation and interview with some sources. They were headman of Ngrendeng, 1 land and farm machines owner, and 5 farmers in Ngrendeng, Ngawi.

\section{B. Methods}

Researcher used Job Hazard Analysis (JHA) form and electronic transcriptions to record source's answer. Their information became justification for validating data using triangulation method. This research was a surveillance using semi-quantitative method and descriptive analysis using AS/NZS 4360:2004 about Risk Management as standard. Research was held in Ngrendeng Village, Ngawi on November 14th-23th 2012.Variables that were measured include hazard, risk, exposure, probability, consequences, basic risk, existing risk, redictive risk, risk control, communication and consultation, and monitoring and review (Fig. 1).

\section{Data Analysis}

Risk was procured from calculation among exposure (E), probability (P), and consequences (C) which were determined from Fine criteria. Basic risk was calculated from formula below.

$$
\text { Risk }=P \times E \times C
$$

Then Existing Risk and Predictive Risk were calculated using formula below.

$$
\text { Risk }=P o F \times E \times C o F
$$

Existing risk is risk level that procured from measuring existing control which has been done by farmers. And predictive risk is risk level that procured from measuring control actions which recommended if those will be done. Final risk level from this research was classification based on risk value. It classified to five levels: high risk $(>350)$, priority 1 (181-350), substantial (71-180), priority 3 (21-70), and acceptable $(\leqslant 20)$.

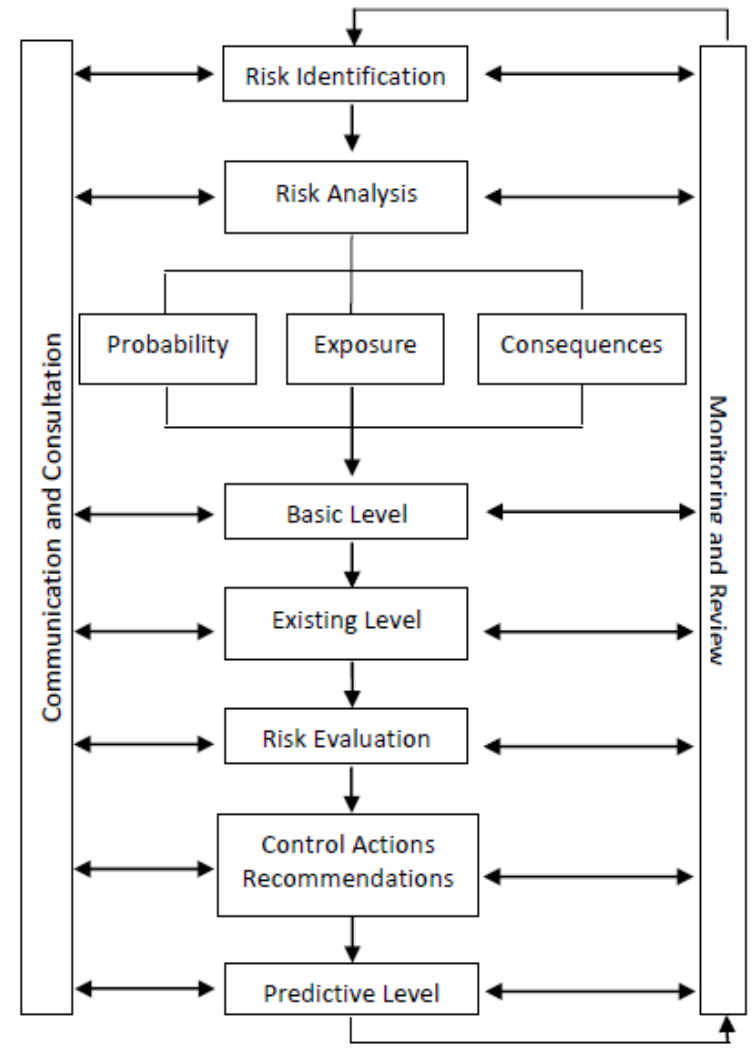

Fig. 1 Conceptual Methodology of Research

\section{RESUlt AND DisCUSSION}

Overall, hazards came from production processes and workplace. Farming processes consist of 13 activities (Fig.2). Other hazard sources that were analyzed include workplace (wet rice field), health behaviour, and work organization and work culture. Total hazards in activities and workplace was 71 risks. The highest risk had value of 1500 (very high) and found in 17 risks (Table. 1). These highest risk could be categorized into six groups. They were noise, vibration, chemical, ergonomic, UV radiation, and health behaviour. Noise and vibration exposure came from vehicles, such as motorcycle, car, tractor, and grinding machine.

Farmers could be exposed for 8 to 10 hours every day with noise exposure of more than $85 \mathrm{dBA}$ and Whole Body Vibration (WBV). This long-time noise exposure may cause Noise Induce Hearing Loss (NIHL). WBV may cause blood and joint disorders, central nerve disorders, and metabolic disorders. Chemical hazard came from exhaust fume and dust from vehicles and road, contact with fertilizer and pesticides (dermal, eye contact, ingestion, inhalation). The farmers were exposed from inhalation, ingestion, and dermal contact. Farmers can suffer respiratory diseases, cancer, nausea, vomiting, headache, eye and skin irritation, central and peripheral nerve disorders, Parkinson's disease, toxication, and disability. 


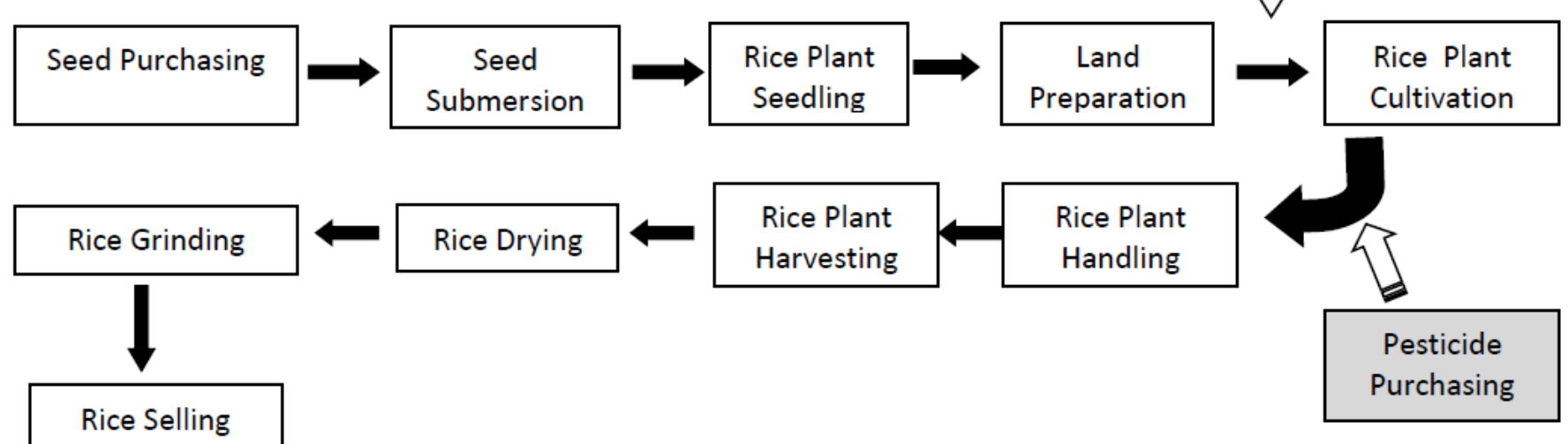

Fig. 2 Rice Farming Processes

TABLE I

CALCULATION OF THE HIGHEST RISK GROUPS

\begin{tabular}{|c|c|c|c|c|c|}
\hline Hazard & Source & Health and Safety Effect & $\begin{array}{c}\text { Basic Risk } \\
\text { Level }\end{array}$ & $\begin{array}{c}\text { Existing Risk } \\
\text { Level }\end{array}$ & $\begin{array}{l}\text { Predictive } \\
\text { Risk Level }\end{array}$ \\
\hline \multirow[t]{2}{*}{ Noise } & Vehicles & $\begin{array}{l}\text { Communication disturbance, } \\
\text { uncomfortable, hearing disorders }\end{array}$ & $\begin{array}{c}1500 \\
\text { (very high) }\end{array}$ & $\begin{array}{c}1500 \\
\text { (very high) }\end{array}$ & $\begin{array}{c}300 \\
\text { (priority 1) }\end{array}$ \\
\hline & Diesel machine (grinding) & $\begin{array}{l}\text { Communication disturbance, } \\
\text { uncomfortable, accident, NIHL }\end{array}$ & $\begin{array}{c}1500 \\
\text { (very high) }\end{array}$ & $\begin{array}{c}1500 \\
\text { (very high) }\end{array}$ & $\begin{array}{c}33.75 \\
\text { (priority 3) }\end{array}$ \\
\hline \multirow[t]{2}{*}{ Vibration } & Vehicles & $\begin{array}{c}\text { Fatigue, uncomfortable, blood } \\
\text { vessel and joint disorders, central } \\
\text { nerve disorders, metabolic disorders }\end{array}$ & $\begin{array}{c}1500 \\
\text { (very high) }\end{array}$ & $\begin{array}{c}1500 \\
\text { (very high) }\end{array}$ & $\begin{array}{c}450 \\
\text { (very high) }\end{array}$ \\
\hline & Diesel machine (grinding) & $\begin{array}{l}\text { Headache, sleepy, stomach ache, } \\
\text { vomiting, fatigue, pins and needles, } \\
\text { blood vessel and joint disorders, } \\
\text { central nerve disorders, metabolic } \\
\text { disorders }\end{array}$ & $\begin{array}{c}1500 \\
\text { (very high) }\end{array}$ & $\begin{array}{c}1500 \\
\text { (very high) }\end{array}$ & $\begin{array}{c}60 \\
\text { (priority 3) }\end{array}$ \\
\hline \multirow[t]{5}{*}{ Chemical } & $\begin{array}{c}\text { Exhaust fumes and dust of } \\
\text { vehicles and road }\end{array}$ & $\begin{array}{l}\text { Asphyxiate, cough, respiratory } \\
\text { infections, pulmonary cancer }\end{array}$ & $\begin{array}{c}1500 \\
\text { (very high) }\end{array}$ & $\begin{array}{c}1500 \\
\text { (very high) }\end{array}$ & $\begin{array}{c}300 \\
\text { (priority 1) }\end{array}$ \\
\hline & Exhaust fumes of tractor & $\begin{array}{c}\text { Asphyxiate, headache, vomiting, } \\
\text { cough, respiratory infections, } \\
\text { pulmonary cancer }\end{array}$ & $\begin{array}{c}1500 \\
\text { (very high) }\end{array}$ & $\begin{array}{c}1500 \\
\text { (very high) }\end{array}$ & $\begin{array}{c}300 \\
\text { (priority 1) }\end{array}$ \\
\hline & $\begin{array}{l}\text { Dental contact with } \\
\text { fertilizer }\end{array}$ & $\begin{array}{l}\text { Eyes irritation, itchiness, espiratory } \\
\text { irritation, laryngeal cancer }\end{array}$ & $\begin{array}{c}1500 \\
\text { (very high) }\end{array}$ & $\begin{array}{c}1500 \\
\text { (very high) }\end{array}$ & $\begin{array}{c}90 \\
\text { (substantial) }\end{array}$ \\
\hline & $\begin{array}{l}\text { Contact with fertilizer } \\
\text { (dermal, eyes, ingestion) }\end{array}$ & $\begin{array}{l}\text { Dermal and eye irritation, itchiness, } \\
\text { vomiting, sore throat, blurred } \\
\text { vision, shaking, headache, sweat, } \\
\text { poisoned, cancer, central and } \\
\text { peripheral nerve disorders, } \\
\text { Parkinson's Disease, disabilities }\end{array}$ & $\begin{array}{c}1500 \\
\text { (very high) }\end{array}$ & $\begin{array}{c}1050 \\
\text { (very high) }\end{array}$ & $\begin{array}{c}42 \\
\text { (priority 3) }\end{array}$ \\
\hline & $\begin{array}{c}\text { Exhaust fumes, grinding } \\
\text { dust and rice husks }\end{array}$ & $\begin{array}{c}\text { Asphyxiate, vomiting, headache, } \\
\text { cough, respiratory infections, } \\
\text { pulmonary cancer }\end{array}$ & $\begin{array}{c}1500 \\
\text { (very high) }\end{array}$ & $\begin{array}{c}1050 \\
\text { (very high) }\end{array}$ & $\begin{array}{c}84 \\
\text { (substantial) }\end{array}$ \\
\hline \multirow[t]{3}{*}{ Ergonomy } & $\begin{array}{l}\text { Squatting while } \\
\text { revoking seed }\end{array}$ & $\begin{array}{l}\text { Foot and waist exhausted, pins and } \\
\text { needles, muscle and bone disorders }\end{array}$ & $\begin{array}{c}1500 \\
\text { (very high) }\end{array}$ & $\begin{array}{c}1500 \\
\text { (very high) }\end{array}$ & $\begin{array}{c}225 \\
\text { (priority 1) }\end{array}$ \\
\hline & Hoeing repetitively & $\begin{array}{l}\text { Fatigue (hand-arm, foot, shoulder, } \\
\text { waist), muscle and spine disorders, } \\
\text { Cumulative Trauma Disorders } \\
\text { (CTDs) }\end{array}$ & $\begin{array}{c}1500 \\
\text { (very high) }\end{array}$ & $\begin{array}{c}1500 \\
\text { (very high) }\end{array}$ & $\begin{array}{c}45 \\
\text { (priority 3) }\end{array}$ \\
\hline & $\begin{array}{l}\text { Walking while } \\
\text { controlling tractor }\end{array}$ & $\begin{array}{c}\text { Limbs exhaustion, muscle and joint } \\
\text { disorders }\end{array}$ & $\begin{array}{c}1500 \\
\text { (very high) }\end{array}$ & $\begin{array}{c}600 \\
\text { (very high) }\end{array}$ & $\begin{array}{c}30 \\
\text { (priority 3) }\end{array}$ \\
\hline
\end{tabular}




\begin{tabular}{|c|c|c|c|c|c|}
\hline & $\begin{array}{l}\text { Blending down and } \\
\text { walking backward }\end{array}$ & $\begin{array}{l}\text { Limbs exhaustion, backache, } \\
\text { muscle and joint disorders }\end{array}$ & $\begin{array}{c}1500 \\
\text { (very high) }\end{array}$ & $\begin{array}{c}600 \\
\text { (very high) }\end{array}$ & $\begin{array}{c}30 \\
\text { (priority 3) }\end{array}$ \\
\hline & Bearing sprayer & $\begin{array}{c}\text { Hand-arm and shoulder exhaustion, } \\
\text { backache, muscle and joint } \\
\text { disorders, CTDs }\end{array}$ & $\begin{array}{c}1500 \\
\text { (very high) }\end{array}$ & $\begin{array}{c}1500 \\
\text { (very high) }\end{array}$ & $\begin{array}{c}37,5 \\
\text { (priority 3) }\end{array}$ \\
\hline & $\begin{array}{l}\text { Bending down while } \\
\text { harvesting repetitively }\end{array}$ & $\begin{array}{l}\text { Limbs exhaustion, fatigue, muscle } \\
\text { and joint disorders, CTDs }\end{array}$ & $\begin{array}{c}1500 \\
\text { (very high) }\end{array}$ & $\begin{array}{c}1500 \\
\text { (very high) }\end{array}$ & $\begin{array}{c}75 \\
\text { (substantial) }\end{array}$ \\
\hline UV Radiation & Sun's rays & $\begin{array}{l}\text { Irritation, sunburned, erythema, } \\
\text { dehydration, cataract, dermal cancer }\end{array}$ & $\begin{array}{c}1500 \\
\text { (very high) }\end{array}$ & $\begin{array}{c}600 \\
\text { (very high) }\end{array}$ & $\begin{array}{c}168 \\
\text { (substantial) }\end{array}$ \\
\hline Health Behaviour & Smoking while farming & $\begin{array}{l}\text { Asthma, cough, respiratory } \\
\text { infections, Cardio Vascular Diseace } \\
\text { (CVD), hypertension, cancer }\end{array}$ & $\begin{array}{c}1500 \\
\text { (very high) }\end{array}$ & $\begin{array}{c}1500 \\
\text { (very high) }\end{array}$ & $\begin{array}{c}450 \\
\text { (very high) }\end{array}$ \\
\hline
\end{tabular}

Ergonomic hazard comes from various awkward postures when farmers work. The postures of interest includes quatting, repetitive hoeing, bending down, controlling vehicle, bearing, etc. These posture might cause muscle exhaustion, muscle and joint disorders, spine disorders, and Cumulative Trauma Disorders (CTDs). Sun's rays was the source of UV radiation. Farmers were exposed for 8 to 10 hours every day. UV radiation may cause irritation, erythema, cataract, dehydration, and skin cancer. Health behaviour that was high risk was smoking. Farmers usually smoke while working. This behaviour may cause cough, respiratory diseases, cardiovascular disease, hypertension, and cancer. Basic risk level consisted of 36 high risks, 6 priority 1risks, 13 substantial risks, 12 priority 3 risks, and 4 acceptable risks. Then all existing control in each risk was analyzed so the existing risk level could be procured. Existing risk level consisted of 33 high risks, 4 priority 1risks, 18 substantial risks, 10 priority 3 risks, and 6acceptable risks. After that, all risk was analyzed and recommendation was made to minimize the risk. Risk level was calculated again to produce predictive risk level. Final predictive risk level consisted of 2 high risks, 8 priority 1 risks, 16 substantial risks, 23 priority 3 risks, and 22acceptable risks. Comparison of risks distribution is showed on fig. 3 .

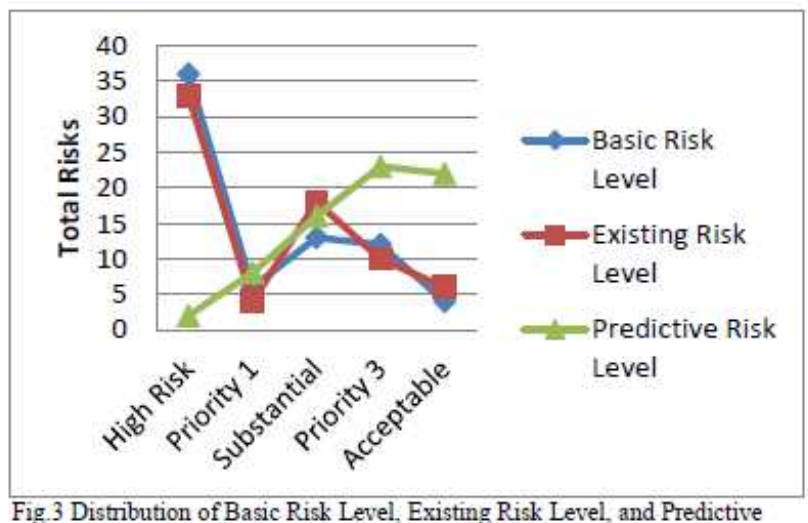
Risk Level in rice farmers' activities

Communication and consultation occured among farmers group in each Ngrendeng sub-village. These groups' activities included routine forum twice a month, information sharing about agriculture engineering and training that was provided by Government. Government programs that had been held in Ngrendeng were counseling and agricultural aid. The aid included seed, fertilizer, and pesticides. Nevertheless, this process could not be said that was done because this process did not involve any concerned authorities, such as Agricultural Ministry, Health Ministry, Labour and Transmigration Ministry, farmers, and businessmen. Risk assessment, which had been made, should be communicated to all stakeholders through government meeting, counseling, promotion, and information media such as leaflet, module, and poster. All concerned authorities should have integrated agricultural program. Monitoring and review were rarely held by Government. Though this processes were held, those were limited to agricultural activities only, not included labour and OHS problems. Government authorities should arrange integrated and sinergic development program immediately. All farmers activities both indoor or outdoor should be monitored so the Government can take immediate action if there is any inexpediencies. Moreover, all activities should be reviewed routinely to minimize in effectivity and inefficiency.

\section{CONCLUSIONS}

Rice farming activities consisted of 16 tasks which had 71 total risks. Grinding activities was activity with the highest risk level. The highest risks from whole process had the value of 1500 (very high), they included noise exposure(vehicle, tractor, grinding machine), vibration exposure(vehicle, grinding machine), UV radiation (sun's rays), ergonomic (squatting, repetitive hoeing, bending down, controlling vehicle, bearing, etc), chemical (exhaust fume,dust, hull of rice), and health behaviour (smoking). In general, risk management process in agricultural activitieswhich includes risk identification, risk assessment, risk control, communication and consultation, and monitoring and review, has not been well done.Rice farming was an agriculture activity with highrisks that harmful for farmers' health and safety. Therefore, OHS program would be needed to manage hazard and risk and keep farmers' productivities.

\section{RECOMMENDATIONS}

Risk management study that has been made should be informed to all rice farmers through farmers group forum that was held in Ngrendeng to become the reference in 
establishing integrated and sinergic development programs among Government concerned authorities. Implementation of routine counseling about agricultural health and safety should be initiated by community leaders through farmers meeting forum and OHS implementation modules for farmers. Moreover, there is urgency to arrange general procedure regarding work organization that all farmers agreed to. Some control actions that need to informed to farmers are using long-sleeve clothes, trousers, hat, and boots if possible, stretching and resting, mineral water consumption, providing first aid, engineering control on tractor and grinding machine, such as make stirrup on tractor, enclosure, longer exhaust pipe design, etc.

\section{ACKNOWLEDGMENT}

The author wish to thank our colleagues from Faculty of Public Health Universitas Indonesia for the knowledge, thoughtful review, and helpful comments.

\section{REFERENCES}

[1] The Health and Safety Executive website. [Online]. Available: http://www.hse.gov.uk/agriculture/hsagriculture.htm.

[2] Afriyanto, "Kajian Keracunan Pestisida pada Petani PenyemprotCabe di Desa Candi Kecamatan Bandungan Kabupaten Semarang,"M. Environmental Health. Thesis. Universitas Diponegoro,Semarang, Indonesia. 2008.

[3] (2012) Centers for Disease Control and Prevention website. [Online]. Available: http://www.cdc.gov/niosh/topics/aginjury/.

[4] Benjamin O. Alli, Fundamental Principles of Occupational Health and Safety, Geneva, Switzerland: ILO, 2001.

[5] Australian Government, Identifying Hazards in the Workplace,Canberra, Australia: Comcare, 2004.

[6] "Statistik Indonesia 2011," Badan Pusat Statistik, Indonesia.

[7] Bureau for Workers'Activities International Labour Office, YourHealth and Safety at Work: A Collection of Modules Controlling Hazards, Geneva, Switzerland: ILO, 1996.

[8] The Health and Safety Executive website. Available: http://www.hse.gov.uk/agriculture/resources/coshh/index.htm.

[9] Tracey J. Dickson, "Calculating risks: fine's mathematical formula 30years later," Australian Journal Outdoor Education. 2001.Available:http://www.freepatentsonline.com/article/AustralianJournal- Outdoor-Education/159791018.html.

[10] International Labour Office, Code of Practice on Safety and Healthin Agriculture, Geneva, Switzerland: ILO, 2010.
[11] International Labour Office, Global Employment Trends 2012,Geneva, Switzerland: ILO, 2012.

[12] Unang G. Kartasasmita and Sumarno. "Kemelaratan bagi PetaniKecil di Balik Kenaikan Produktivitas Padi," Sinar Tani, ed. 30, pp. Dec. 2009.

[13] Dilshad Ahmed Khan et al, "Risk Assessment of Pesticide Exposureon Health of Pakistani Tobacco Farmers," Journal of Exposure Science and Environmental Epidemiology, vol. 20.

[14] L. Meily Kurniawidjaja, Teori dan Aplikasi Kesehatan Kerja,Jakarta, Indonesia: UI Press, 2010

[15] Soekidjo Notoatmodjo, Metodologi Penelitian Kesehatan, Jakarta,Indonesia: Rineka Cipta, 2010.

[16] ---------------------. Promosi Kesehatan dan Ilmu Perilaku, Jakarta,Indonesia: Rineka Cipta, 2007.

[17] Ilham Nyak et al, "Laporan Akhir Penelitian TA 2007: Analisis ProfilPetani dan Pertanian Indonesia," BPPT Departemen Pertanian, Jakarta, 2007.

[18] Ngrendeng RPJM Arranger Committee, "Rencana PembangunanJangka Menengah Desa Tahun 2009-2014," Ngrendeng, Ngawi, 2009.

[19] Pertanian Sehat Indonesia. (2012) "Pentingnya Analisis ResiduPestisida." [Online]. Available:http://pertaniansehat.com/read/2012/08/09/pentingnyaanalisisresidu-pestisida.html.

[20] Peraturan Menteri Pertanian Nomor 1 Tahun 2007, AgriculturalMinistry, 2007.

[21] Barbara A. Plog and Patricia J. Quinlan, Fundamentals of IndustrialHygiene, United States of America: National Safety Council, 2002.

[22] Soehatman Ramli, Pedoman Praktis Manajemen Risiko dalamPerspektif K3, Jakarta, Indonesia: Dian Rakyat, 2010.

[23] Kathrine Rugbjerg et al, "Pesticide Exposure and Risk of Parkinson'sDisease - A Population-Based Case - Control Study Evaluating ThePotential for Recall Bias," Scandinavia Journal of Work, Environment and Health, vol. 5, pp. 37, Sept. 2011.

[24] John Ridley, Ikhtisar Kesehatan dan Keselamatan Kerja, Jakarta,Indonesia: Erlangga, 2008.

[25] Risk Management AS/NZS 4360:2004. Australia Standards \& NewZealand Standards, 2004.

[26] Sarah Elizabeth Starks, "Neurological Outcomes Among PesticidesApplicators," Ph.D Epidemiology Dissertation, The University of Iowa, Iowa, USA, Dec. 2010.

[27] "Survei Angkatan Kerja Nasional 2004-2011," Badan Pusat Statistik, Jakarta,Indonesia.Available:http://www.bps.go.id/tab_sub/view.php? kat=1\&tabel=1\&daftar=1\&id_subyek=06\&notab $=2$.

[28] J.B. Temperley and L. J. Fragar, Farm Worker Safety Induction Manual 1: Program and Principles, Sydney, Australia: Australian Center for Agricultural Health and Safety University of Sydney, Oct.2009.

[29] World Health Organization, IPCS Risk Assessment Terminology,Geneva, Switzerland: WHO, 2004 